\author{
Wei-Tien Chang \\ Matthew Huei-Ming Ma \\ Kuo-Liong Chien \\ Chien-Hua Huang \\ Min-Shan Tsai \\ Fuh-Yuan Shih \\ Ann Yuan \\ Kuang-Chau Tsai \\ Fang-Yue Lin \\ Yuan-Teh Lee \\ Wen-Jone Chen
}

\section{Postresuscitation myocardial dysfunction: correlated factors and prognostic implications}

Received: 28 March 2006

Accepted: 6 October 2006

Published online: 15 November 2006

(C) Springer-Verlag 2006

Electronic supplementary material This article has been selected for the initiative "Publishing the Review Process", and the material related to its review process can be obtained on-line as Electronic Supplementary Material using the Springer Link server located at http:// dx.doi.org/10.1007/s00134-006-0442-9 and is accessible for authorized users.

W.-T. Chang · M. H.-M. Ma · C.-H. Huang · M.-S. Tsai - F.-Y. Shih · A. Yuan · K.-C. Tsai · W.-J. Chen ( $)$

National Taiwan University Hospital and National Taiwan University College of Medicine, Department of Emergency Medicine,

Chung-Shan South Road 7, 100 Taipei,

R.O.C.

e-mail: jone@ha.mc.ntu.edu.tw

Tel.: +886-2-23562168

Fax: +886-2-23223150

W.-T. Chang - M. H.-M. Ma - K.-L. Chien · C.-H. Huang · Y.-T. Lee · W.-J. Chen National Taiwan University Hospital and National Taiwan University College of Medicine, Division of Cardiology, Department of Internal Medicine, Chung-Shan South Road 7, 100 Taipei, R.O.C.

\section{F.-Y. Lin}

National Taiwan University Hospital and National Taiwan University College of Medicine, Division of Cardiovascular Surgery, Department of Surgery, Chung-Shan South Road 7, 100 Taipei, R.O.C.

\section{K.-L. Chien}

College of Public Health, National Taiwan University, Institute of Preventive Medicine, Taipei, R.O.C.

Abstract Objective: To evaluate the clinical factors correlated with postresuscitation myocardial dysfunction and the prognostic implication such dysfunction may have. Design and setting: Prospective observational study in a university medical center Patients: 58 adult patients successfully resuscitated from nontraumatic out-of-hospital cardiac arrest over 2 years. Measments and results: Echocardiographic evaluation of the left ventricular systolic and diastolic functions was performed $6 \mathrm{~h}$ postresuscitation and was analyzed in correlation to the clinical features and resuscitation factors. Univariate analysis revealed left ventricular ejection fraction (LVEF) to be significantly lower in patients with hypertension, past history of myocardial infarction, resuscitation duration longer than 20 min, defibrillation, and use of more than $5 \mathrm{mg}$ epinephrine. Isovolumic relaxation time (IVRT) was significantly longer in patients with noncardiac cause and initial rhythm of nonventricular fibrillation/tachycardia. Multiple regression analysis showed epinephrine dose and past history of myocardial infarction to be independent factors for LVEF, while the cause of cardiac arrest was independently associated with IVRT. For prognosis, 27 patients survived to hospital discharge. Both LVEF under $40 \%$ and IVRT $100 \mathrm{~ms}$ or longer were associated with poor survival outcomes. In Cox regression analysis IVRT $100 \mathrm{~ms}$ or longer served as an independent factor predicting poor survival prognosis. Conclusions: Postresuscitation left ventricular dysfunction is correlated with a number of clinical factors, among which past history of myocardial infarction, epinephrine dose, and the cause of cardiac arrest play independent roles. Meanwhile, IVRT $100 \mathrm{~ms}$ or longer $6 \mathrm{~h}$ postresuscitation predicts poor survival outcomes and serves as a marker of poor prognosis.

Keywords Cardiac arrest - Cardiopulmonary resuscitation - Postresuscitation - Myocardial dysfunction Survival · Prognosis 


\section{Introduction}

Sudden cardiac arrest has long been an important issue with tremendous socioeconomic impact. In the United States more than 450,000 persons collapse suddenly outside the hospital every year [1]. With advances in emergency cardiac care some $20-40 \%$ of these patients can be rescued and regain spontaneous circulation. However, up to $50-75 \%$ of those initially resuscitated eventually die during hospitalization [2]. The majority of these deaths are due to circulatory or neurological failure, these being the main clinical features of postresuscitation syndrome [3, 4]. Circulatory failure usually occurs early after resuscitation and may lead to multiple organ dysfunction and early mortality. Being the extended link in the chain of survival, early postresuscitation care should focus more on improving hemodynamics and preventing circulatory failure. Postresuscitation myocardial dysfunction therefore becomes a crucial problem to deal with in the early postresuscitation phase.

Postresuscitation myocardial dysfunction has been an entity of increasing importance in the past decade not only for its prognostic impact but also for the interest in its pathogenic mechanisms. In contrast to regional myocardial stunning due to variable periods of coronary occlusion, postresuscitation myocardial dysfunction has been regarded as a specific model of global myocardial stunning resulting from total circulatory arrest followed by resuscitation [5, 6, 7]. Experimental studies have implicated a number of factors in its severity, such as ischemic duration, electrical defibrillation, the number, type, and waveforms of electrical shock, and the dose of epinephrine $[8,9,10,11,12,13,14]$. Efforts have also been made to improve such dysfunction via pharmacological or mechanical means $[15,16,17,18,19,20,21]$. In the real world, although researchers have sought to characterize postresuscitation myocardial dysfunction in humans [22, 23], few have evaluated the factors associated with its occurrence and severity. Its prognostic value also remains largely unknown. While defining these factors may implicate the possible ways of preventing or treating the myocardial dysfunction after resuscitation, it should be valuable to figure them out. This prospective study was thus conducted aiming at evaluating the factors associated with postresuscitation myocardial dysfunction and the prognostic implications that it may confer.

\section{Materials and methods}

This was a prospective observational study evaluating postresuscitation myocardial function in the clinical settings. The collection of data and echocardiographic evaluation of the patients were conducted in accordance with the Declaration of Helsinki and with institutional guidelines. The approval of the study protocol was obtained from the Medical Ethics Committee of the University Hospital.

\section{Patient selection}

Between 1 January 2001 and 31 December 2002 were enrolled consecutively 337 adult patients ( $>18$ years) successfully resuscitated from nontraumatic out-of-hospital cardiac arrest in a prospective registry at a university tertiary medical center. After return of spontaneous circulation (ROSC) the patients were admitted to intensive care units for postresuscitation care. ROSC was achieved in 130 patients (39\%), and 93 (28\%) survived to admission to the intensive care unit; 35 of these died in the subsequent hours. Finally, 58 patients $(17 \%)$ were included when echocardiography was performed $6 \mathrm{~h}$ after resuscitation (Table 1). Cardiopulmonary resuscitation (CPR) procedures were performed according to ACC/AHA guidelines. After ROSC postresuscitation care was basically supportive, using a standardized protocol in the hospital. Vasopressor support was instituted if hypotension occurred in the postresuscitation phase. The dose was continuously adjusted to maintain systolic blood pressure at least $90 \mathrm{mmHg}$. No specific intervention such as hypothermia was employed during the study period. Left ventricular (LV) regional wall motion abnormalities were noted in 13 patients, including 10 with acute myocardial infarction (MI) as the cause of cardiac arrest and 3 with medical past history of MI. In these patients LVEF was measured using the area-length method.

\section{Data collection}

Relevant information including patient characteristics, medical past history, prehospital care data, and resuscitation factors were collected. The prehospital care data were obtained by review of the Taipei Out-of-hospital Cardiac Arrest Registry Form, an Utstein-based registry of the city [24]. Variables included the time from cardiac arrest to start of CPR, the durations of basic and advanced life support, use of automated external defibrillator, and any drugs used in the prehospital settings. The initial cardiac rhythm was classified as ventricular fibrillation/tachycardia (VF/VT), asystole, and pulseless electrical activities. Of the 24 patients (41\%) receiving defibrillation 16 presented with VF/VT. The remaining 8 with initial rhythm of asystole or pulseless electrical activity developed VF/VT during CPR, requiring the use of defibrillation. For patients receiving defibrillation during CPR, the number and energy of shock were recorded. The vasopressors (dopamine and/or norepinephrine) employed in the early postresuscitation phase were also recorded. Epinephrine was employed in 54 patients (93\%); in 4 cases ROSC was achieved after defibrillation 
Table 1 Clinical information of the 58 patients (IQR interquartile range, $C P R$ cardiopulmonary resuscitation, $R O S C$ return of spontaneous circulation)

\begin{tabular}{lc}
\hline Mean age (years; IQR) & $73.5(61.8-83.3)$ \\
Male sex & $36(62 \%)$ \\
Medical past history & \\
Hypertension & $33(56.9 \%)$ \\
Ischemic heart disease & $17(29.3 \%)$ \\
Old myocardial infarction & $9(15.5 \%)$ \\
Cardiac cause & $29(50 \%)$ \\
Myocardial ischemia/infarction & $19(33 \%)$ \\
Acute myocardial infarction & $10(17 \%)$ \\
Ischemia-related cardiac arrhythmia & $9(16 \%)$ \\
Hypertrophic cardiomyopathy & $4(7 \%)$ \\
Others & $6(10 \%)$ \\
Time factors (min; IQR) & \\
No CPR duration & $10.0(5.0-20.0)$ \\
Total CPR time & $19.5(10.0-31.3)$ \\
Basic life support & $3.0(0-15.0)$ \\
Advanced cardiac life support & $11.5(8.0-22.0)$ \\
Total time from arrest to ROSC & $33.5(20.8-53.5)$ \\
Initial cardiac rhythm & \\
Ventricular fibrillation/tachycardia & $16(28 \%)$ \\
Asystole & $28(48 \%)$ \\
Pulseless electrical activity & $14(24 \%)$ \\
Defibrillation & \\
Automated external defibrillator & $15(26 \%)$ \\
Manual defibrillation at emergency room & $9(15 \%)$ \\
Total energy delivered (J; IQR) & $860(263-1865)$ \\
Epinephrine & \\
Employed during CPR & $54(93 \%)$ \\
Median dose (mg; IQR) & $4.0(3.0-8.0)$ \\
Inotropic agents used in early & \\
postresuscitation phase & \\
Dopamine & $26(45 \%)$ \\
Dopamine + norepinephrine & $16(27 \%)$ \\
\hline
\end{tabular}

and/or CPR without the need of epinephrine. In the early postresuscitation phase vasopressors were employed in 42 cases (26 with dopamine and 16 with dopamine plus norepinephrine) when echocardiography was performed. None of the patients received dobutamine or intra-aortic balloon counterpulsation. The cause of cardiac arrest was determined by the physician-in-charge, and was further reviewed by the study physicians. If there was controversy, this was settled after discussion among the physicians.

\section{Echocardiographic measurements}

With patients in left decubitus position, transthoracic echocardiography was performed $6 \mathrm{~h}$ postresuscitation by an experienced cardiologist blinded to the patient's detailed clinical information. Using a GE-Vingmed System 5 device with a $2.5-$ to $4.5-\mathrm{MHz}$ transducer, all measurements were made according to the recommendations of the American Society of Echocardiography [25, 26]. Using $M$ mode echocardiography, the internal diameters of the LV chambers, interventricular septum, and posterior wall were obtained from the parasternal long-axis view, both at end-diastolic and end-systolic phases. The LV dimensions were divided by the body surface area and expressed as index values. The LV ejection fraction (LVEF) was calculated using Teichholz' method. In patients with LV regional wall motion abnormalities, the area-length method was substituted. To determine LV diastolic function we measured isovolumic relaxation time (IVRT) from aortic valve closure to mitral valve opening using pulsed-wave Doppler echo.

\section{Outcome evaluation}

Patients' survival outcome was monitored continuously after ROSC. In those successfully discharged the neurological outcome was determined at hospital discharge using the cerebral performance categories (CPC) score [24]. Good neurological recovery was defined as CPC score of 1. Twenty-seven patients (8\%) survived to hospital discharge. Of these $11(3 \%)$ had good neurological recovery with CPC score of 1 . The other 16 patients $(5 \%)$ were discharged with impaired neurological function (one in CPC 2, one in CPC 3, and 14 in CPC 4). The duration of hospitalization ranged from 0.3 to 72 days, with a median of 11 days.

\section{Statistical analysis}

Statistical analysis used SAS version 9.1 and STATA version 9. Considering the nonnormal distribution of the variables, univariate analysis of the factors associated with postresuscitation LV function was carried out by Mann-Whitney/Wilcoxon test. The significant factors identified in the univariate analysis were then analyzed in a multiple regression model. For survival prognosis we used the log-rank test to compare LV functions and other relevant clinical factors. Those found significant were then entered for Cox regression analysis. The neurological outcomes were also analyzed in correlation with these factors, after which multiple logistic regression analysis was performed to determine independent factors.

\section{Results}

Factors correlated with postresuscitation LV dimensions and functions

The associations between clinical factors and postresuscitation LV dimensions and functions are shown in Table 2. Both LV end-diastolic (LVEDDi) and end-systolic diameter index values (LVESDi) were significantly higher in patients with cardiac cause and past history of ischemic heart disease and MI. LVESDi was also higher in patients receiving defibrillation or at least $5 \mathrm{mg}$ epinephrine. For 
Table 2 Factors associated with postresuscitation left ventricular dimensions and functions. ( $L V E D D$ left ventricular end-diastolic diameter, $L V E S D$ left ventricular end-systolic diameter, $L V E F$ left ventricular ejection fraction, IVRT isovolumic relaxation time, $C P R$ cardiopulmonary resuscitation, $V F / V T$ ventricular fibrillation/ventricular tachycardia, $A E D$ automated external defibrillator)

\begin{tabular}{|c|c|c|c|c|}
\hline & LVEDD index $\left(\mathrm{mm} / \mathrm{m}^{2}\right)$ & LVESD index $\left(\mathrm{mm} / \mathrm{m}^{2}\right)$ & $\operatorname{LVEF}(\%)$ & IVRT (ms) \\
\hline \multicolumn{5}{|l|}{ Sex } \\
\hline Male $(n=36)$ & $28.2(24.3-31.8)$ & $19.7(16.5-24.2)$ & $50.0(38.5-62.8)$ & $90.0(80.0-110.0)$ \\
\hline Female $(n=22)$ & $26.5(23.5-30.8)$ & $17.3(14.7-23.8)$ & $60.0(46.5-74.0)$ & $70.0(80.0-112.5)$ \\
\hline \multicolumn{5}{|l|}{ Age } \\
\hline$\geq 75$ years old $(n=26)$ & $25.3(21.9-31.9)$ & $17.4(14.6-24.2)$ & $55.0(41.0-66.5)$ & $85.0(70.0-110.0)$ \\
\hline$<75$ years old $(n=32)$ & $28.2(25.3-30.9)$ & $20.1(16.0-23.5)$ & $54.0(40.0-65.8)$ & $90.0(80.0-117.5)$ \\
\hline \multicolumn{5}{|l|}{ Past history of hypertension } \\
\hline Yes $(n=33)$ & $27.4(23.0-33.6)$ & $21.2(15.2-26.1)$ & $48.0(31.5-61.5)$ & $80.0(70.0-115.0)$ \\
\hline No $(n=25)$ & $27.9(24.0-30.1)$ & $17.3(15.4-20.0)$ & $60.0(49.5-70.0) *$ & $90.0(80.0-110.0)$ \\
\hline \multicolumn{5}{|c|}{ Past history of ischemic heart disease } \\
\hline Yes $(n=17)$ & $33.1(27.5-37.6)$ & $23.7(16.1-29.9)$ & $52.0(31.5-62.5)$ & $70.0(80.0-95.0)$ \\
\hline No $(n=41)$ & $26.5(28.1-23.6)^{* * *}$ & $18.2(14.7-21.2)^{*}$ & $57.0(41.0-68.0)$ & $90.0(80.0-120.0)$ \\
\hline \multicolumn{5}{|c|}{ Past history of old myocardial infarction } \\
\hline Yes $(n=9)$ & $33.5(26.5-37.6)$ & $27.6(19.2-32.9)$ & $48.0(26.5-54.0)$ & $80.0(70.0-95.0)$ \\
\hline No $(n=49)$ & $27.4(23.9-29.1)^{*}$ & $18.2(15.0-21.5)^{*}$ & $58.0(42.0-68.0)^{*}$ & $90.0(80.0-110.0)$ \\
\hline \multicolumn{5}{|l|}{ Cause } \\
\hline Cardiac $(n=29)$ & $28.8(24.4-36.3)$ & $20.9(15.5-28.8)$ & $51.0(31.5-65.0)$ & $80.0(70.0-90.0)$ \\
\hline Noncardiac $(n=29)$ & $26.5(22.9-28.6)^{*}$ & $17.4(14.7-21.1)^{*}$ & $59.0(45.5-67.0)$ & $100.0(85.0-125.0)^{* * *}$ \\
\hline \multicolumn{5}{|l|}{ No CPR duration } \\
\hline$\geq 10 \min (n=33)$ & $28.2(24.8-31.0)$ & $19.6(17.0-24.2)$ & $55.0(44.0-68.0)$ & $90.0(80.0-115.0)$ \\
\hline$<10 \min (n=25)$ & $26.5(21.2-31.5)$ & $18.2(14.8-22.2)$ & $55.0(35.0-65.5)$ & $80.0(70.0-100.0)$ \\
\hline \multicolumn{5}{|l|}{ Total CPR time } \\
\hline$\geq 20 \min (n=29)$ & $25.9(22.4-32.1)$ & $18.5(15.1-24.3)$ & $49.0(31.0-62.0)$ & $90.0(70.0-105.0)$ \\
\hline$<20 \min (n=29)$ & $28.1(25.6-31.0)$ & $19.9(15.8-22.5)$ & $60.0(47.0-68.0) *$ & $90.0(80.0-115.0)$ \\
\hline \multicolumn{5}{|l|}{ Initial cardiac rhythm } \\
\hline VF/VT $(n=16)$ & $28.2(23.4-35.4)$ & $20.1(16.0-26.4)$ & $50.5(38.5-64.5)$ & $80.0(70.0-90.0)$ \\
\hline Non-VF/VT $(n=42)$ & $27.4(24.0-30.3)$ & $18.3(15.0-23.8)$ & $56.0(41.5-66.5)$ & $95.0(80.0-120.0) * *$ \\
\hline \multicolumn{5}{|l|}{ Defibrillation during CPR } \\
\hline Yes $(n=24)$ & $28.6(24.4-33.4)$ & $20.5(16.9-27.3)$ & $47.0(33.3-59.8)$ & $80.0(70.0-90.0)$ \\
\hline No $(n=34)$ & $26.8(23.5-29.4)$ & $17.4(14.6-21.4)^{*}$ & $59.0(45.8-70.0)^{*}$ & $95.0(80.0-120.0)$ \\
\hline \multicolumn{5}{|l|}{ Dose of epinephrine } \\
\hline$\geq 5 \mathrm{mg}(n=23)$ & $28.7(23.1-33.1)$ & $20.0(17.4-26.3)$ & $46.0(37.0-55.0)$ & $90.0(80.0-100.0)$ \\
\hline$<5 \mathrm{mg}(n=35)$ & $27.4(24.3-30.2)$ & $17.3(14.1-21.9)^{*}$ & $60.0(45.0-70.0)^{* *}$ & $90.0(80.0-120.0)$ \\
\hline \multicolumn{5}{|c|}{ Use of vasopressors in the postresuscitation phase } \\
\hline Yes $(n=42)$ & $27.7(23.5-30.3)$ & $18.2(14.9-23.8)$ & $55.0(39.5-65.3)$ & $90.0(80.0-110.0)$ \\
\hline No $(n=16)$ & $27.8(24.4-33.6)$ & $20.1(15.4-27.0)$ & $51.5(41.3-73.0)$ & $85.0(62.5-117.5)$ \\
\hline
\end{tabular}

$* p=<0.05, * * p=<0.01, * * * p=<0.001$

LV systolic function, poorer LVEF was associated with hypertension, past history of MI, CPR duration longer than $20 \mathrm{~min}$, defibrillation, and the use of at least $5 \mathrm{mg}$ epinephrine. For LV diastolic function, IVRT was significantly longer in patients with noncardiac cause and initial rhythm of non-VF/VT. For patients with myocardial ischemia/infarction as the cause of cardiac arrest, no significant differences were noted in LV dimensions or functions compared to other cardiogenic patients without evident myocardial ischemia. Multiple regression analysis showed past history of ischemic heart disease to be significantly associated with LVEDDi while epinephrine dose and history of ischemic heart disease were independent factors for LVESDi (Table 3). For LV systolic function, epinephrine dose and past history of MI were significantly associated with LVEF. For diastolic function, the cause of cardiac arrest was independently associated with IVRT (Table 3).
Table 3 Clinical factors associated with the LV dimensions and functions in multiple regression analysis $(C I$ confidence interval, $L V E D D$ left ventricular end-diastolic diameter, $L V E S D$ left ventricular end-systolic diameter, $L V E F$ left ventricular ejection fraction, IVRT Isovolumic relaxation time)

\begin{tabular}{llll}
\hline & $\begin{array}{l}\text { Estimated } \\
\text { parameter }\end{array}$ & $95 \%$ CI & $p$ \\
\hline $\begin{array}{l}\text { LVEDD index }\left(\mathrm{mm} / \mathrm{m}^{2}\right) \\
\quad \text { Past history of ischemic } \\
\text { heart disease }\end{array}$ & 6.2 & 3.0 to 9.4 & $<0.001$ \\
$\begin{array}{l}\text { LVESD index }\left(\mathrm{mm} / \mathrm{m}^{2}\right) \\
\quad \text { Past history of ischemic } \\
\text { heart disease }\end{array}$ & 6.6 & 3.0 to 10.3 & 0.001 \\
$\quad \begin{array}{l}\text { Epinephrine dose }(\geq 5 \mathrm{mg}) \\
\text { LVEF }(\%)\end{array}$ & 4.7 & 1.3 to 8.1 & 0.008 \\
$\quad \begin{array}{l}\text { Past history of } \\
\text { myocardial infarction }\end{array}$ & -15.6 & $-28.1 \rightarrow 3.1$ & 0.015 \\
$\quad \begin{array}{l}\text { Epinephrine dose }(\geq 5 \mathrm{mg}) \\
\text { IVRT (ms) } \\
\quad \text { Cause: cardiac }\end{array}$ & -12.8 & $-22.0 \rightarrow 3.6$ & 0.007 \\
\hline
\end{tabular}


Prognostic implications of postresuscitation LV dysfunction

Log-rank tests revealed both LVEF and IVRT to be significantly associated with patient survival outcome $(p=0.03$ and 0.0004 , respectively). The results are demonstrated by Kaplan-Meier survival curves in Fig. 1. Other factors associated with survival included age, initial cardiac rhythm, epinephrine dose, and total CPR duration. After adjusting for these variables in Cox regression analysis, IVRT longer than $100 \mathrm{~ms}$ still served as an independent predictor for poor survival prognosis (hazard ratio 3.3, 95\% confidence interval 1.6-6.7, $p=0.002$ ). Neurological recovery was significantly better in patients with LVEF greater than $40 \%$ ( $24 \%$ vs. $0 \%$ in LVEF $<40 \%, p=0.04$ ), but this relationship no longer reached statistical significance in multiple regression analysis. Factors independently predicting neurological recovery were initial rhythm of VF/VT, dura-
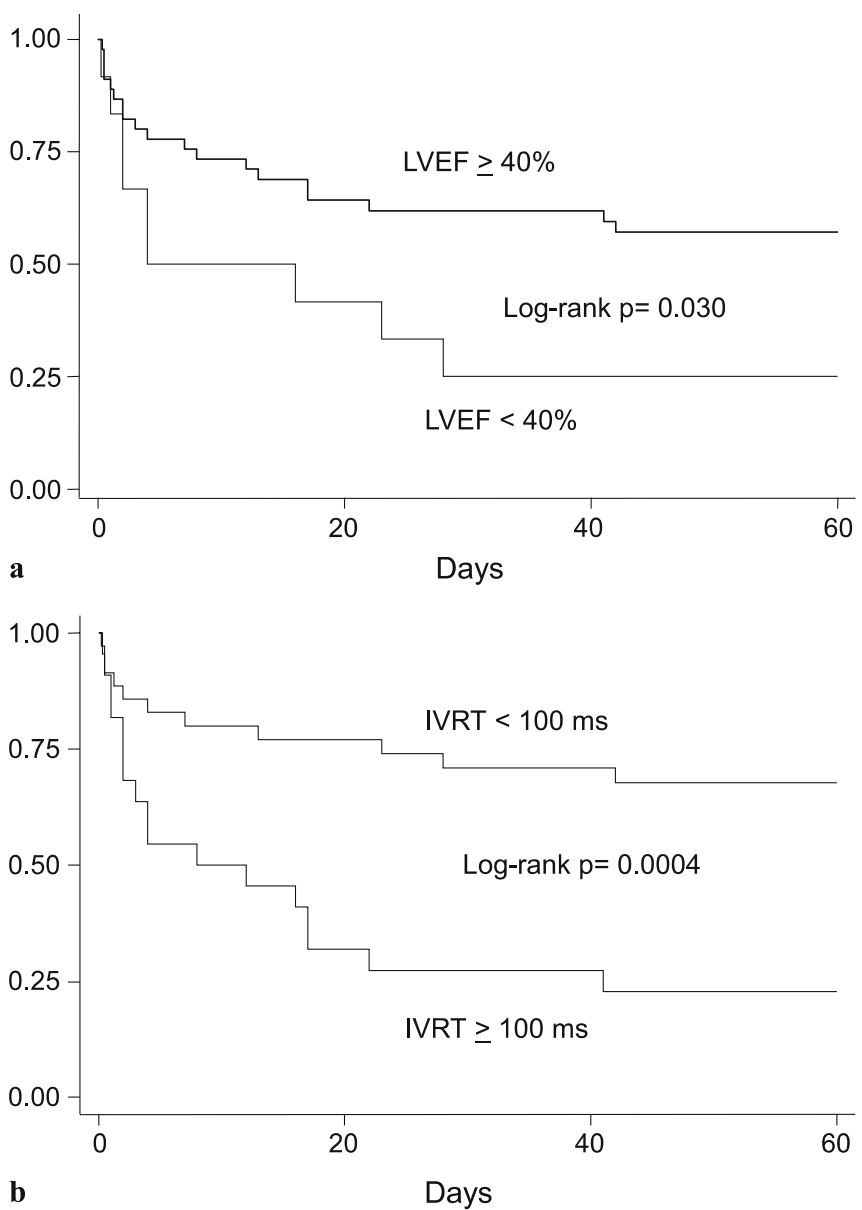

Fig. 1 Kaplan-Meier survival curves of the patients stratified by left ventricular ejection fraction (a) and isovolumic relaxation time (b) tion of no CPR less than 10 min, and total CPR time less than $20 \mathrm{~min}$.

\section{Discussion}

Postresuscitation myocardial dysfunction is a specific model of global ischemia and reperfusion. In contrast to experimental animal studies in which cardiac arrest is artificially induced in previously healthy animals, the scenarios are usually more complicated in the real world. Cardiac arrest may result from a variety of pathologies in patients with diversified underlying diseases. Postresuscitation myocardial dysfunction may be a combination of the patient's prearrest conditions and those resulting from cardiac arrest and CPR. Most of all, these patient and resuscitation factors often interact with one another, making their roles difficult to verify. To date only few human studies have focused on evaluating the factors contributing to postresuscitation myocardial dysfunction. This clinical study therefore may provide valuable information based on which further preventive measures and treatments can be developed.

Factors associated with postresuscitation LV dimensions

In the failing heart the ventricular chamber tends to dilate when the myocardium fails to effectively pump out the blood. This has been described in animal resuscitation studies in which the LV dilates in the early postresuscitation phase and then recovers within 2-3 days when myocardial contractility resumes $[6,7]$. Clinically the factors influencing LV size are more complicated. Both the patient's underlying heart diseases and resuscitation factors affect LV size measured during the postresuscitation phase. In this study LV dimensions were significantly larger in patients with cardiac cause and past histories of ischemic heart disease and old MI. Meanwhile, those receiving a larger dose of epinephrine also had higher LVESDi. While larger LV chamber might reflect the patient's preexisting heart diseases, which in turn leads to cardiogenic cardiac arrest and need for more epinephrine during resuscitation, multiple regression analysis identified the history of ischemic heart disease as an independent factor for LVEDDi and LVESDi. This suggests that LV size during the postresuscitation phase reflects underlying conditions more than the effects arising from cardiac arrest and CPR. Nevertheless the fact that epinephrine dose also serves as an independent factor for LVESDi suggests that resuscitation factors may at least in part affect LV size during the early postresuscitation phase. This is especially true when higher LVESDi indeed represents failure of the myocardium to effectively pump out the blood, a result 
consistent with the fact that higher epinephrine dose is associated with lower LVEF (Table 3).

Factors associated with postresuscitation LV systolic dysfunction

Resuscitation factors such as longer CPR duration, defibrillation, and higher cumulative dose of epinephrine were found to be negatively associated with postresuscitation LV systolic function. Similarly, LVEF was also poorer in patients with past histories of hypertension and old MI. Although the associations with CPR duration and defibrillation were consistent with a variety of animal studies and clinical observations $[9,10,11,12,27,28,29]$, multiple regression analysis suggested that past history of MI and epinephrine dose play more independent roles. The former represents the result of an old insult to the myocardium. The pathogenic mechanism of epinephrine deserves more elaboration. Although being formally recommended in various resuscitation emergencies, epinephrine has been found to adversely affect the postresuscitation myocardial function [30,31]. This clinical study further supports such notion by showing high epinephrine dose as an independent factor of postresuscitation myocardial dysfunction. One possible mechanism is that, following large dose of epinephrine, marked hypertension and tachycardia in the immediate postresuscitation phase remarkably increase LV afterload and worsen the balance between oxygen supply and myocardial demand [32, 33]. Recent studies in molecular cardiology also suggest that catecholamine itself may induce apoptosis in cardiomyocyte via $\beta$-adrenergic and caspase pathways $[34,35]$. Although the need for higher epinephrine dose during CPR may reflect the poorer underlying cardiac function, all such evidence shed light on the pathognomonic properties epinephrine may have during and after CPR.

Factors associated with postresuscitation LV diastolic function

For LV diastolic function a number of parameters have been used to determine ventricular compliance and active relaxation. Many of these, however, are inevitably influenced by factors such as preload and afterload. Among these parameters the time constant of isovolumic-pressure decline $(\tau)$ is less influenced by such factors and has often been used in animal studies. However, as this parameter is obtained via invasive monitoring, it seems less practical in clinical practice. Echocardiographic measurement of IVRT proves to be an ideal substitute for $\tau$ since it is measured noninvasively and has been shown to be well correlated with $\tau$ [36]. IVRT may become prolonged if active relaxation of the left ventricle is impaired; in contrast, it may be shortened with impaired ventricular compliance. In this study IVRT was significantly longer in patients with noncardiac causes and initial rhythm of non-VF/VT. The former also served as an independent factor for IVRT. While postresuscitation LVEF was not significantly different between patients with cardiac and noncardiac causes, the distinct discrimination in IVRT suggests different pathogenic processes along the course of cardiac arrest and CPR.

Prognostic value of the postresuscitation LV dysfunction

Postresuscitation LV dysfunction with circulatory failure has been considered closely correlated with early mortalities despite initial success in resuscitation. Laurent et al. [23] in their clinical hemodynamic evaluation found that persistent low cardiac index $24 \mathrm{~h}$ postresuscitation was associated with multiple organ failure and early death. In this study we demonstrated the prognostic value of postresuscitation myocardial dysfunction at an even earlier stage. Both LVEF less than $40 \%$ and IVRT $100 \mathrm{~ms}$ or longer $6 \mathrm{~h}$ after resuscitation predictied poorer survival outcomes. Furthermore, IVRT longer than $100 \mathrm{~ms}$ was an independent prognostic indicator after adjusting for other resuscitation factors. These findings are of clinical importance since earlier identification of myocardial problem is crucial before hemodynamics finally decline. Therefore it is advisable for clinicians to evaluate LV functions in the early postresuscitation hours for guiding further resuscitation and critical care. Moreover, the potentials of improving prognosis by treatments aimed at improving postresuscitation myocardial dysfunction should be explored. This would be most important for those with severe postresuscitation LV dysfunction.

For neurological outcomes, although LVEF greater than $40 \%$ was associated with better neurological recovery, this factor no longer existed in multiple regression analysis. This result is consistent with previous observation by Laurent et al. [23] suggesting that postresuscitation myocardial dysfunction and neurological injury are both consequences of the more decisive factors such as the duration of ischemic insult. Postresuscitation myocardial dysfunction itself does not directly impact the neurological outcomes. However, while myocardial dysfunction may worsen global circulation and hence brain perfusion, it should still be worthwhile to test whether treatment and improvement in postresuscitation myocardial dysfunction enhance the neurological prognosis.

\section{Limitations}

There are a few limitations in this study. First, only fewer than one-half (58/130) of the initially resuscitated patients survived to the 6th $\mathrm{h}$ and were included. LV function may 
be even worse in the nonincluded population. The original design of echocardiographic evaluation at $6 \mathrm{~h}$ instead of immediate postresuscitation was based on previous studies $[5,6,7,23]$ and on our own clinical observations suggesting that myocardial dysfunction is most pronounced $6 \mathrm{~h}$ after CPR. It was also to avoid the remnant epinephrine effect that may mask myocardial dysfunction in the immediate po stresuscitation period. However, myocardial dysfunction may develop soon after cardiac arrest and sometimes during the CPR sequence [37]. Clinically we did see a number of patients exhibiting severe LV dysfunction immediately after CPR, almost all of whom died soon after due to profound shock. This nonstudied population may have even more severe postresuscitation myocardial dysfunction and graver prognosis.

Second, while vasopressors were often used in the early postresuscitation phase, it might have affected the ventricular function measured. Nevertheless, since this study is aimed at evaluating postresuscitation myocardial dysfunction in the real world, demonstration of the ventricular dysfunction under regular clinical practice is reasonable. It would be difficult and unethical if vasopressors were discontinued only for LV function measurement. Meanwhile, it is not hard to imagine an even worse prognosis if the ventricular function is severely compromised even under strong inotropic support. Third, the diagnosis of cardiac arrest cause was mostly clinical based rather than pathologically confirmed. Although this is generally acceptable in clinical studies since autopsy are usually unavailable, there are chances when clinical diagnosis fails to represent the real pathology.

\section{Conclusions}

In conclusion, postresuscitation myocardial dysfunction is associated with a number of clinical factors, including the patient's underlying conditions and those related to resuscitation. Among these, past history of MI and the dose of epinephrine are negatively correlated with LV systolic function, while the cause of cardiac arrest serves as an independent factor for diastolic dysfunction. Most of all, IVRT measured $6 \mathrm{~h}$ postresuscitation provides independent, valuable prognostic implications. Based on these observations it should be worthwhile to launch multicenter prospective studies combining echocardiography and other imaging modalities for better characterizing postresuscitation myocardial dysfunction and stratifying the patients at risk. Clinical trials assessing treatment modalities aiming at improving myocardial function postresuscitation should also be explored for overcoming the critical phase of circulatory failure early after resuscitation.

Acknowledgements. This study was supported by a study grant 90N-016 from National Taiwan University Hospital, Taipei, Taiwan, R.O.C. The authors thank all medical and nursing staffs involved in the CPR and postresuscitation care of the patients.

\section{References}

1. Gillum RF (1989) Sudden coronary death in the United States, 1980-1985. Circulation 79:756-765

2. Kern KB (2002) Postresuscitation myocardial dysfunction. Cardiol Clin 20:89-101

3. Schoenenberger RA, von Planta M, von Planta I (1994) Survival after failed out-of-hospital resuscitation. Arch Intern Med 154:2433-2437

4. Laver S, Farrow C, Turner D, Nolan J (2004) Mode of death after admission to an intensive care unit following cardiac arrest. Intensive Care Med 30:2126-2128

5. Tang W, Weil MH, Sun S, Gazmuri RJ, Bisera J (1993) Progressive myocardial dysfunction after cardiac resuscitation. Crit Care Med 21:1046-1050

6. Gazmuri R, Weil MH, Bisera J, Tang W, Fukui M, McKee D (1996) MyoCardial dysfunction after successful resuscitation from cardiac arrest. Crit Care Med 24:992-1000
7. Kern KB, Hilwig RW, Rhee KH, Berg RA (1996) MyoCardial dysfunction after resuscitation from cardiac arrest: an example of global myocardial stunning. J Am Coll Cardiol 28:232-240

8. Plamer BS, Hadziahmetovic M, Veci T, Angelos MG (2004) Global ischemic duration and reperfusion function in the isolated perfused rat heart. Resuscitation 62:97-106

9. Yamaguchi $\mathrm{H}$, Weil $\mathrm{MH}$, Tang $\mathrm{W}$, Kamohara T, Jin X, Bisera J (2002) MyoCardial dysfunction after electrical defibrillation. Resuscitation 54:289-296

10. Xie J, Weil MH, Sun S, Tang W, Sato Y, Jin X, Bisera J (1997) Highenergy defibrillation increases the severity of postresuscitation myocardial dysfunction. Circulation 96:683-688

11. Gazmuri RJ, Deshmukh S, Shah PR (2000) MyoCardial effects of repeated electrical defibrillation in the isolated fibrillating rat heart. Crit Care Med 28:2690-2696
12. Tang W, Weil MH, Sun S, Yamaguchi H, Povoas HP, Pernat AM, Bisera J (1999) The effects of biphasic and conventional monophasic defibrillation on postresuscitation myocardial function. J Am Coll Cardiol 34:815-822

13. Tang W, Weil MH, Sun S, Jorgenson D, Morgan C, Klouche K, Synder D (2004) The effects of biphasic waveform design on post-resuscitation myocardial function. J Am Coll Cardiol 43:1228-1235

14. Tang W, Weil MH, Sun S, Noc M, Yang L, Gazmuri RJ (1995) Epinephrine increases the severity of postresuscitation myocardial dysfunction. Circulation 92:3089-3093

15. Kern KB, Hilwig RW, Berg RA, Rhee KH, Sanders AB, Otto CW, Ewy GA (1997) Postresuscitation left ventricular systolic and diastolic dysfunction: treatment with dobutamine. Circulation 95:2610-2613 
16. Zhao L, Zhang W, Zhang W, Zheng Y (2000) The effects of high-dose epinephrine combined with isoprenaline on isolated rabbit heart and cardiomyocytes after cardioversion of ventricular fibrillation. Mol Cell Biochem 207:71-75

17. Angelos MG, Murray HN, Waite MD, Gorsline RT (2002) Postischemic inotropic support of the dysfunctional heart. Crit Care Med 30:410-416

18. Tang W, Well MH, Sun S, Pernat A, Mason E (2000) $K_{\text {ATP }}$ channel activation reduces the severity of postresuscitation myocardial dysfunction. Am J Physiol Heart Circ Physiol 279:1609-1615

19. Zaugg CE, Ziegler A, Lee RJ, Barbosa V, Buser PT (2002) Postresuscitation stunning: postfibrillatory myocardial dysfunction caused by reduced myofilament $\mathrm{Ca}^{2+}$ responsiveness after ventricular fibrillation-induced myocyte $\mathrm{Ca}^{2+}$ overload. J Cardiovasc Electrophysiol 13:1017-1024

20. Tennyson H, Kern KB, Hilwig RW, Berg RA, Ewy GA (2002) Treatment of postresuscitation myocardial dysfunction: aortic counterpulsation versus dobutamine. Resuscitation 54:69-75

21. Vasquez A, Kern KB, Hilwig RW, Heidenreich J, Berg RA, Ewy GA (2004) Optimal dosing of dobutamine for treating post-resuscitation left ventricular dysfunction. Resuscitation 61:199-207

22. Mullner M, Domanovits H, Sterz F, Herkner H, Gamper G, Kurkciyan I, Laggner AN (1998) Measment of myocardial contractility following successful resuscitation: quantitated left ventricular systolic function utilizing non-invasive wall stress analysis. Resuscitation 39:51-59

23. Laurent I, Monchi M, Chiche JD, Joly LM, Spaulding C, Bourgeois B, Cariou A, Rozenberg A, Carli P, Weber S, Dhainaut JF (2002) Reversible myocardial dysfunction in survivors of out-of-hospital cardiac arrest. J Am Coll Cardiol 40:2110-2116
24. Cummins RO, Chamberlain DA, Abramson NS, Allen M, Baskett PJ, Becker L, Bossaert L, Delooz HH, Dick WF, Eisenberg MS (1991) Recommendation guidelines for uniform reporting of data from out-of-hospital cardiac arrest: the Utstein style. A statement for health professionals from a task force of the American Heart Association, the European Resuscitation Council, the Heart and Stroke Foundation of Canada, and the Australian resuscitation Council. Circulation 84:960-975

25. Sahn D, DeMaria A, Kisslo J, Weyman A (1978) Recommendations regarding quantitation in M-mode echocardiography: results of a survey of echocardiographic measurements. Circulation 58:1072-1077

26. Schiller NB, Shah PM, Crawford M, Demaria A, Devereux R, Feigenbaum H, Gutgesell H, Reichek N, Sahn D, Schnittger I (1989) Recommendations for quantitation of the left ventricle by two-dimensional echocardiography. J Am Soc Echocardiogr 2:358-367

27. Stiell IG, Wells GA, Hebert PC, Laupacis A, Weitzman BN (1995) Association of drug therapy with survival in cardiac arrest: limited role of advanced cardiac life support drugs. Acad Emerg Med 2:264-273

28. Layon AJ, Gabrielli A, Goldfeder BW, Hevia A, Idris AH (2003) Utstein style analysis of rural out-of-hospital cardiac arrest [OOHCA]: total cardiopulmonary resuscitation time inversely correlates with hospital discharge rate. Resuscitation 56:59-66

29. Niemann JT, Garner D, Lewis RJ (2003) Left ventricular function after monophasic and biphasic waveform defibrillation: the impact of cardiopulmonary resuscitation time on contractile indices. Acad Emerg Med 10:9-15

30. Roberts D, Landolfo K, Dobson K, Light RB (1990) The effects of methoxamine and epinephrine on survival and regional distribution of cardiac output in dogs with prolonged ventricular fibrillation. Chest 98:999-1005
31. Tang W, Weil MH, Sun S, Noc M, Yang L, Gazmuri RJ (1995) Epinephrine increases the severity of postresuscitation myocardial dysfunction. Circulation 92:3089-3093

32. Berg RA, Otto CW, Kern KB, Sanders AB, Hilwig RW, Hansen KK, Ewy GA (1994) High-dose epinephrine results in greater early mortality after resuscitation from prolonged cardiac arrest in pigs, a prospective, randomized study. Crit Care Med 22:282-290

33. Ditchey RV, Lindenfeld J (1988) Failure of epinephrine to improve the balance between myocardial oxygen supply and demand during closed-chest resuscitation in dogs. Circulation 78:382-389

34. Communal C, Singh K, Pimentel DR, Colucci WS (1998) Norepinephrine stimulates apoptosis in adult rat ventricular myocyte by activation of the beta-adrenergic pathway. Circulation 98:1329-1334

35. Fu YC, Chi CS, Yin SC, Hwang B, Chiu YT, Hsu SL (2004) Norepinephrine induces apoptosis in neonatal rat cardiomyocytes through a reactive oxygen species-TNF $\alpha$ caspase signaling pathway. Cardiovasc Res 62:558-567

36. Schober KE, Fuentes VL, Bonagura JD (2003) Comparison between invasive hemodynamic measurements and noninvasive assessment of left ventricular diastolic function by use of Doppler echocardiography in healthy anesthetized cats. Am J Vet Res 64:93-103

37. Klouche K, Weil MH, Sun S, Tang W, Povoas HP, Kamohara T, Bisera J (2002) Evolution of the stone heart after prolonged cardiac arrest. Chest 122:1006-1011 